American Journal of Pharmaceutical Education 2019; 83 (8) Article 7083.

\title{
RESEARCH
}

\section{Characteristics of Drug-Related Podcasts and This Medium's Potential as a Pharmacy Education Tool}

\author{
Sean P. Kane, PharmD, ${ }^{a}$ Michael Shuman, PharmD, ${ }^{a}$ Khyati Patel, PharmD, ${ }^{a}$ Margaret Olson, PharmD ${ }^{b}$ \\ ${ }^{a}$ Rosalind Franklin University of Medicine and Science, North Chicago, Illinois \\ ${ }^{\mathrm{b}}$ Presence Saints Mary and Elizabeth Medical Center, Chicago, Illinois \\ Submitted March 26, 2018; accepted October 8, 2018; published October 2019.
}

Objective. To analyze the publication frequency and characteristics of drug-related podcasts and describe the role of pharmacists in creating content for this audio-based educational medium.

Methods. Podcasts that potentially included drug-related educational information were identified based on four podcast categories that were publicly available as of June 2016. Podcasts were screened by two reviewers to determine whether they contained at least five episodes with drug-related content and a minimum of 10 audio episodes. Metrics related to the podcast, audio episodes, and names of the content authors were collected and a descriptive analysis was conducted.

Results. Of the 960 podcasts screened, 125 met the study criteria and were included in the descriptive analysis. These drug-related podcasts produced a median of two episodes per month and each episode lasted an average of 27 minutes. The most common professions represented by podcast hosts and guests were physicians $(83.2 \%)$, followed by nurses and nurse practitioners $(11.2 \%)$ and pharmacists $(10.4 \%)$. Podcast content varied widely, with critical care and emergency medicine being the most common (18.4\%), followed by general medicine (14.4\%) and complementary and alternative medicine (13.6\%). Conclusion. Drug-related podcasts are numerous and easily accessible; however, the quality and accuracy of their content cannot be easily determined as episodes do not consistently cite references. Pharmacists appear to be underrepresented in developing this particular genre of educational content, pharmacy educators should consider producing and disseminating educational material through podcasts for students and practitioners.

Keywords: podcast, pharmacist, technology, audio, education

\section{INTRODUCTION}

Podcasting has become a popular medium for sharing information, including educational content. A podcast is a digital audio file published on an internet platform, such as iTunes (Apple Inc., Cupertino, CA), and made available for listeners to download at their convenience using software on a computer or a mobile device. ${ }^{1}$ With the growth of podcast apps, identifying relevant podcasts and downloading audio content has become very easy and requires minimal technical knowledge. As a teaching tool, podcasts can be used to review complex topics and encourage lifelong learning. Podcasts can even be used as assigned content for a flipped classroom teaching approach. ${ }^{2}$

The number of podcasts published online more than doubled between 2012 and $2015 .{ }^{3}$ Furthermore, the num-

Corresponding Author: Sean P. Kane, Department of Pharmacy Practice, Rosalind Franklin University of Medicine and Science, 3333 Green Bay Rd., North Chicago, IL 60064. Tel: 847-578-7630. Fax: 847-775-6593. E-mail:

sean.kane@rosalindfranklin.edu ber of monthly podcast listeners has grown by $75 \%$ since 2013, indicating the acceptance of podcasting as a means of content delivery. The growth and simplicity of podcast production has resulted in potentially unreliable or inappropriate content being produced, particularly with topics related to health professions education. As there is no central authority to review and approve podcasts and their content, even authors with little or no health care background are able to publish podcasts on medical topics. In addition, literature analyzing the prevalence of medical podcasts is scarce and what has been published is now outdated given the growth in podcasting. ${ }^{1,4}$

Pharmacists play a key role in the dissemination of drug-related information and education to other health care providers, students, and patients. While drug-related podcasts are produced by various health care providers, pharmacists appear to be underrepresented as podcast authors. The objective of this study was to analyze the frequency of publication and characteristics of drug-related podcasts and to describe the authors' professional training 


\section{American Journal of Pharmaceutical Education 2019; 83 (8) Article 7083.}

with a particular focus on the role of pharmacists in creating this audio-based educational content.

\section{METHODS}

This study sought to include all drug-related podcasts published in the iTunes (US) store as of June 14, 2016. An initial list of all potential podcasts was generated by collecting the top 240 podcasts from each of four iTunes categories (medicine, social sciences, higher education, and alternative health) that were deemed potentially relevant to drug-related topics. This resulted in a list of 960 podcasts, which was then further assessed.

Podcasts were included in the study if they had at least 10 audio episodes publicly available at the time of review and at least five of those were drug-related. A drug-related episode was defined as audio content that discussed a drug or class of drugs, using the Food and Drug Administration's (FDA's) definition of a drug: “a substance intended for use in the diagnosis, cure, mitigation, treatment, or prevention of disease." ${ }^{5}$ For example, an episode discussing ginger as a cooking ingredient would not meet the definition of a drug-related episode, but an episode evaluating the use of ginger in the context of an antiemetic would meet the criteria. Video content was not evaluated and did not contribute toward the minimum requirements for inclusion. Podcasts were excluded if the audio content was not available in English, had a drug-related focus but on non-human subjects (eg, veterinary medicine), or required a fee or password for access.

Each podcast was evaluated for inclusion by a pharmacy faculty member and a pharmacy student in a separate, blinded fashion. Podcasts were immediately included or excluded if both reviewers reached blinded agreement. For podcasts with a discrepancy regarding inclusion or exclusion, all four authors discussed these podcasts until a consensus was reached.

Once a list of podcasts meeting the study criteria was generated, additional information was collected regarding each podcast using both manual data collection and automated, software-based collection from the podcasts' Really Simple Syndication (RSS) data feed. Elements of data collection included podcast information available in iTunes and metrics regarding the frequency of audio episodes and their duration. Information about each podcast's therapeutic content area was collected by reviewing the podcast's description in iTunes and, if necessary, listening to episode content. To collect information about the professional training of the podcast hosts and guests as well as further information regarding episode content, the six most recent audio episodes (up to June 14, 2016), the podcast's website, and the iTunes directory entry were listened to and analyzed. This research involved publicly available data (podcasts) and did not involve the study of human subjects, so institutional review board approval was not required. Descriptive analyses were performed using SPSS version 24 (IBM Corporation, Armonk, NY).

\section{RESULTS}

Of the 960 podcasts identified in the four predetermined iTunes categories, 813 were excluded after a blinded review and agreement between two reviewers. Thirty-three other podcasts were further discussed by all four reviewers to resolve inclusion or exclusion discrepancies (Figure 1). Of these 33 podcasts, an additional 17 were excluded after group consensus. Thus, 130 drug-related podcasts met study inclusion criteria. However, five of these were excluded when it was discovered they had been removed from iTunes, resulting in a final list of 125 podcasts for analysis. The majority of these podcasts originated from authors or organizations in the United States (87.2\%), followed by Australia (4.8\%), Canada (4\%), and the United Kingdom (4\%).

Among the 125 drug-related podcasts, 110 provided sufficient historical data to conduct a descriptive analysis and timeline of previously published episodes. The remaining 15 podcasts only provided a fixed number of recent episodes rather than a complete list of all published episodes. As shown in Figure 2, there was a generally consistent number of new drug-related podcasts produced beginning in 2006 and continuing on for nearly a decade.

Among the 110 podcasts that provided a full set of historical episode data, there was a median of 78 total episodes per podcast (interquartile range $[\mathrm{IQR}]=49-216$ episodes) and a median of two new episodes released per month $(\mathrm{IQR}=1.0-4.1$ episodes $)$. Of the 110 podcasts that

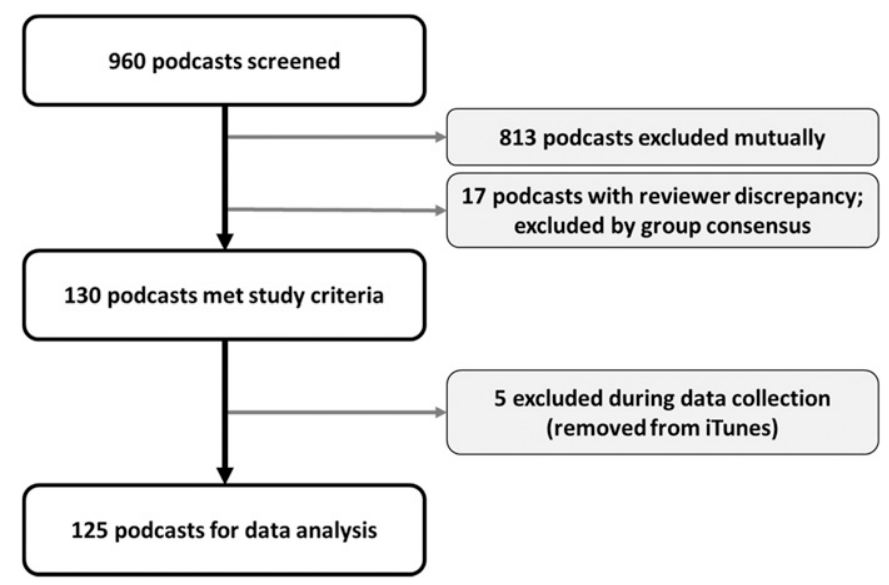

Figure 1. Screening and Inclusion Process for Podcast Episodes 


\section{American Journal of Pharmaceutical Education 2019; 83 (8) Article 7083.}

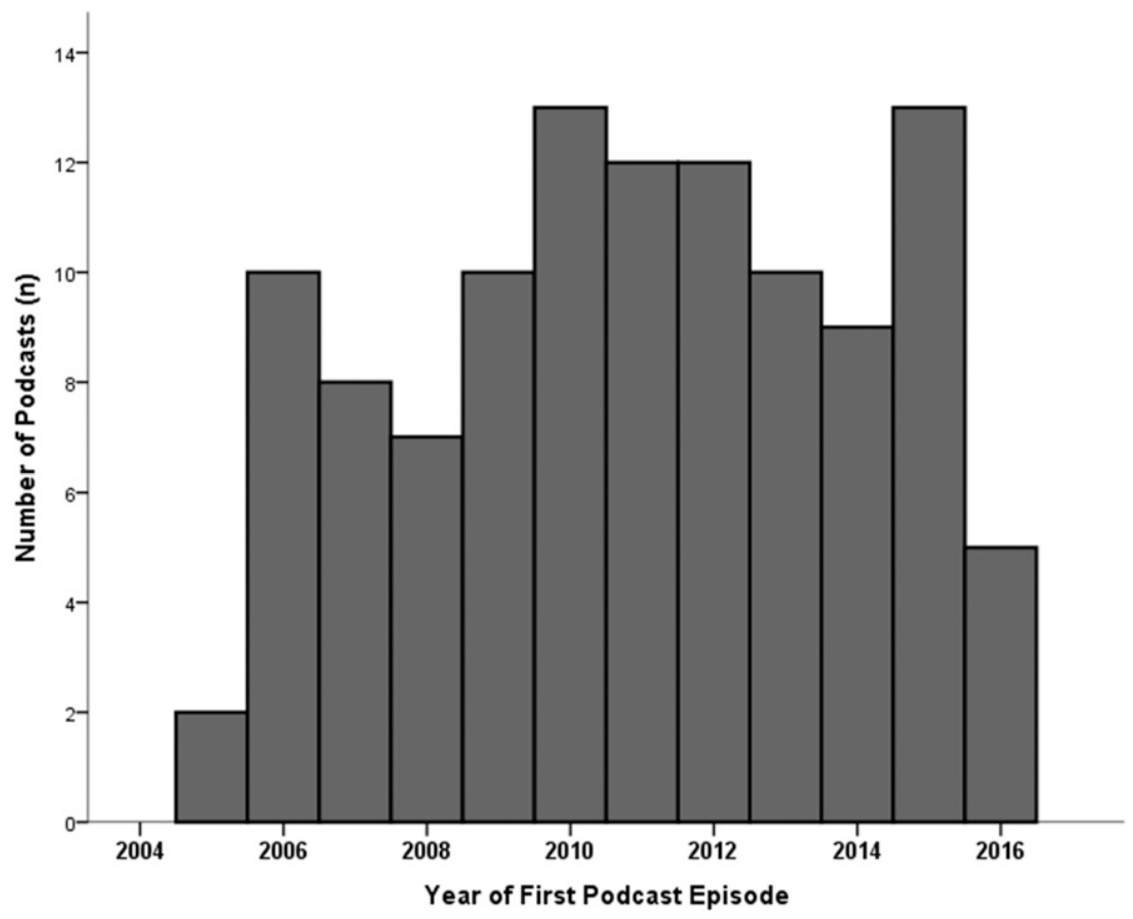

Figure 2. Chronology of Drug-Related Podcast Creation by Year $(n=110)^{\mathrm{a}}$

${ }^{\text {a }}$ Fifteen podcasts did not provide older episodes for adequate analysis.

provided historical episode data, 81 provided average episode length as part of their data feed, with a median episode duration of 27 minutes (IQR $=16-34$ minutes).

Information about the professional training of the host and guests was available for 122 of the 125 podcasts included in the study. As shown in Table 1, the vast majority of podcasts used physicians to provide episode content, followed by nursing professionals. Pharmacists were the third most common health care professionals to provide content, and were represented in only $10.4 \%$ of drugrelated podcasts.

Table 1. Self-Identified Professional Training Among Podcast Hosts and Guests

\begin{tabular}{lc}
\hline $\begin{array}{l}\text { Professional Training of } \\
\text { Host or Guest }\end{array}$ & $\begin{array}{c}\text { All Podcasts } \\
\text { (N=125), No. (\%) }\end{array}$ \\
\hline Physician & $104(83.2)$ \\
Nurse or Nurse Practitioner & $14(11.2)$ \\
Pharmacist & $13(10.4)$ \\
Naturopath & $7(5.6)$ \\
Chiropractor & $6(4.8)$ \\
Physician Assistant & $3(2.4)$ \\
Dietitian & $2(1.6)$ \\
Podiatrist & $1(0.8)$ \\
Psychologist & $1(0.8)$ \\
Physical therapist & $1(0.8)$ \\
Not specified in podcast & $3(2.4)$ \\
\hline
\end{tabular}

With regards to the professional affiliation of the podcasts, $36(28.8 \%)$ were affiliated with a peer-reviewed journal (frequently as a table of contents synopsis) or a professional health care organization. Five podcasts were affiliated with a university or college, two were affiliated with a hospital or medical center, and two were affiliated with a government organization. The majority (64\%) of podcasts had no such affiliation and were frequently produced by individuals.

The general content areas covered by these drug-related podcasts were quite diverse, but as shown in Table 2, the combined topic of critical care and emergency medicine was the most represented, contributing $18.4 \%$ of all included podcasts. General medicine and complementary medicine were the next two most frequent topics, with the top three topic areas accounting for about $50 \%$ of all podcasts in the analysis. In the review of the episode content, $45.6 \%$ of podcasts provided references to peer-reviewed articles to support the material discussed in the audio episodes. Finally, $69.6 \%$ of podcasts were clearly targeted to health care providers, $14.4 \%$ to patients and their caregivers, and $16 \%$ did not specify the intended audience.

\section{DISCUSSION}

Podcasting drug-related information is a unique modality for targeting both the education of pharmacy students and lifelong learning for practicing pharmacists and other health care providers. Especially for the roughly $30 \%$ of students who are auditory learners, the accessibility 


\section{American Journal of Pharmaceutical Education 2019; 83 (8) Article 7083.}

Table 2. General Content Areas of Podcasts Analyzed to Identify Drug-Related Content and the Role of Pharmacists in this Educational Medium

\begin{tabular}{lc}
\hline Content Areas & $\begin{array}{c}\text { All Podcasts } \\
(\mathbf{n = 1 2 5}), \mathbf{N o}(\mathbf{\%})\end{array}$ \\
\hline Critical care and emergency medicine & $23(18.4)$ \\
General medicine (including internal & $18(14.4)$ \\
$\quad$ medicine and family medicine) & \\
Complementary and alternative medicine & $17(13.6)$ \\
Pediatrics (including subspecialties & $9(7.2)$ \\
$\quad$ within pediatrics) & \\
Oncology & $7(5.6)$ \\
Psychiatry and mental health & $7(5.6)$ \\
Infectious diseases & $6(4.8)$ \\
Pharmacy or pharmacology & $6(4.8)$ \\
Neurology & $4(3.2)$ \\
Cardiology & $3(2.4)$ \\
Gastroenterology & $3(2.4)$ \\
Nursing & $3(2.4)$ \\
Obstetrics and gynecology & $3(2.4)$ \\
Other content areas ${ }^{\text {a }}$ & $11(8.8)$ \\
No specific focus to podcast content & $5(4.0)$ \\
\hline
\end{tabular}

${ }^{a}$ Other areas include geriatric medicine, global health, endocrinology, surgery, physician assistant, anesthesia, and basic sciences

and user-friendly nature of podcasts makes podcasts a highly attractive method of learning. ${ }^{1,4}$

To our knowledge, this is the first comprehensive review describing the availability of drug-related podcasts and the professional qualifications of those presenting the content. Previous analyses, published nearly a decade ago, were restricted only to podcasts published by peer-reviewed journals rather than all types of podcasts. A recent review of podcasting in medical education concluded that this modality is feasible and acceptable to medical leaners; however, there is a lack of evidence regarding the efficacy of medical podcasting as a teaching method either as a primary or a supplementary medium for student learning. ${ }^{6}$ Furthermore, there is a lack of data describing the landscape of both health care and drugrelated podcasts, particularly specific to pharmacy education. With the growing popularity of podcasts, including those that contain health care education, there is a need for pharmacy educators to analyze this novel learning modality.

Given that the focus of this analysis was specific to drug-related podcasts, the very low representation of pharmacists $(10.4 \%)$ suggests that pharmacists may not be publishing podcast content as extensively as other health professionals. Combined with the fact that only $45.6 \%$ of the podcasts we reviewed contained references to peer-reviewed publications, this raises the question of the accuracy of the content of these podcasts. Further- more, in nine of the podcasts that provided drug-related information, none of the guests or hosts were health care providers whose roles involved using drug therapy in clinical practice. These podcasts either did not provide sufficient data to describe the professional qualifications of the guests or hosts, had a PhD host without any clinical experience, or had hosts with backgrounds in naturopathic or chiropractic medicine. These concerns are of particular importance as it relates to the education of future pharmacists. Podcasting is an attractive medium for pharmacy students and has been successfully used to supplement lecture material and improve examination scores. ${ }^{7,8}$ It may also help with preparation for experiential rotations or board examinations, as some peer-reviewed journals are now offering article content in podcast format. ${ }^{7}$

This study did not specifically evaluate the quality or accuracy of the podcast material. At least two publications have proposed consensus-based quality indicators, specific to podcasts, with both objective and subjective metrics for assessment. ${ }^{9,10}$ While these quality indicators may exist, there is no specific scoring system or threshold to use to deem a particular podcast is credible or of high quality.

For a pharmacy educator interested in pursuing publication of a podcast as an educational modality, this analysis provides some details regarding the norms of publication length and frequency. Most podcasts published about two episodes per month, with each episode lasting approximately half an hour. This duration is similar to a survey of emergency medicine residents in which $84.6 \%$ of respondents reported an ideal episode duration was less than 30 minutes. ${ }^{11}$ While this may be the typical frequency and duration, there is opportunity for further research regarding whether these are the optimal metrics to promote learning among listeners.

Our study is not without limitations. Attempts to standardize the inclusion criteria for a podcast being "drug-related" were made by using the FDA definition of "drug" and by conducting a blinded review process between two reviewers. However, classifying a podcast according to the criteria may have been somewhat subjective. Some podcasts may have been excluded on the basis that the content discussed did not meet the full FDA definition for drug therapy used in this study.

There were some limitations when evaluating the professional training of the podcast hosts or guest contributors. Instead of collecting degrees achieved (such as a Doctor of Pharmacy or Bachelor of Science in Pharmacy), we collected information regarding professional training (eg, pharmacist). Frequently, hosts and guests had degrees in a variety of fields, such as a $\mathrm{PhD}$ and a Master of Public Health degree, but it was not clear whether these individuals had actual clinical experience in the treatment of patients. Furthermore, some of the speakers in the 


\section{American Journal of Pharmaceutical Education 2019; 83 (8) Article 7083.}

podcasts did not have medical degrees or training and instead had been part of a research team that included physicians or pharmacists. However, the physicians and pharmacists were not present on the podcast. In these situations, the presenter's ability to speak about drug-related topics may have been greater than an individual without similar colleagues. Finally, it was not possible to validate host credentials; therefore, some individuals categorized as having professional training in a particular field either may not actually have had that training or may have no longer been practicing in the area.

Even when classifying providers by level of professional training, it is unwise to assume that one degree confers greater understanding or greater reputation than another. Not every pharmacist is well qualified to speak on the management of hypertension, just as not every physician is equally informed on matters of pharmacology. With a wide variety of author credentials and qualifications to discuss a topic, referencing peer-reviewed publications is particularly important. Fewer than 50\% of podcasts provided references, which can frequently assist in distinguishing fact from opinion and provide listeners with a source of additional educational material.

According to the US Bureau of Labor statistics, the number of employed physicians outnumbers pharmacists in the United States by a factor of about 2.1. ${ }^{12}$ In this study, however, physician hosts or guests on drug-related podcasts outnumbered pharmacists by a factor of eight. Given that the analysis only included drug-related podcasts, pharmacists were underrepresented as a health care profession despite this being a growth area for pharmacy and drug therapy education. For example, the FDA now uses podcasts to update pharmacists on important new drug safety information. ${ }^{13}$ Lack of incentive, time, and skills to produce audio-based learning material, as well as unfamiliarity with the resources required, may be reasons why more pharmacists are not producing podcasts. Many potential solutions exist to increase pharmacist involvement in podcasting. Two recommendations include recognizing podcasting as a scholarly activity for pharmacy faculty members and making faculty training in the use of podcasting as an educational method more available. Developing methods for evaluating podcasts for their use in providing continuing education to practicing health care providers can also attract many experts in the field to produce similar content. This medium may serve not only as a convenient method for meeting continuing education requirements for busy health care providers, but also provide a platform for interprofessional education and collaboration. A framework for starting a podcast geared toward medical education has been recently published and may be used as a guide moving forward. ${ }^{14}$

\section{CONCLUSION}

Drug-related podcasts are numerous and readily available; however, pharmacists appear to have a limited role in developing this particular type of educational content, and existing episodes fail to consistently cite references. Pharmacy educators should consider exploring podcasting as a means for disseminating content to students and for the provision of continuing education.

\section{REFERENCES}

1. Wilson P, Petticrew M, Booth A. After the gold rush? A systematic and critical review of general medical podcasts. JRSM Short Rep. 2009;102(2):69-74. doi:10.1258/jrsm.2008.080245.

2. Boulos MNK, Maramba I, Wheeler S. Wikis, blogs and podcasts: a new generation of Web-based tools for virtual collaborative clinical practice and education. BMC Med Educ. 2006;6:41. doi:10.1186/ 1472-6920-6-41.

3. Vogt N. Podcasting: Fact Sheet. Pew Research Center's Journalism Project. http://www.journalism.org/2016/06/15/ podcasting-fact-sheet/. Published June 15, 2016. Accessed July 5, 2016.

4. Clauson KA, Vidal DM. Overview of biomedical journal podcasts. Am J Health Syst Pharm. 2008;65(22):2155-2158. doi:10.2146/ajhp080180.

5. Center For Drug Evaluation. Drug Approvals and Databases Drugs@FDA Glossary of Terms. https://www.fda.gov/drugs/drugapprovals-and-databases/drugsfda-glossary-terms. Accessed July 5, 2016.

6. Cho D, Cosimini M, Espinoza J. Podcasting in medical education: a review of the literature. Korean J Med Educ. 2017 Dec;29(4):229239. doi:10.3946/kjme.2017.69.

7. Cain J, Fox BI. Web 2.0 and pharmacy education. Am J Pharm Educ. 2009;73(7):Article 120.

8. Meade O, Bowskill D, Lymn JS. Pharmacology podcasts: a qualitative study of non-medical prescribing students' use, perceptions and impact on learning. BMC Med Educ. 2011;11:2. doi:10.1186/1472-6920-11-2.

9. Lin M, Thoma B, Trueger NS, Ankel F, Sherbino J, Chan T. Quality indicators for blogs and podcasts used in medical education: modified Delphi consensus recommendations by an international cohort of health professions educators. Postgrad Med J. 2015;91(1080):546-550. doi:10.1136/postgradmedj-2014-133230. 10. Thoma B, Chan TM, Paterson QS, Milne WK, Sanders JL, Lin M. Emergency medicine and critical care blogs and podcasts: establishing an international consensus on quality. Ann Emerg Med. 2015;66(4):396-402.e4. doi:10.1016/j.annemergmed.2015.03.002.

11. Riddell J, Swaminathan A, Lee M, et al. A survey of emergency medicine residents' use of educational podcasts. West J Emerg Med. 2017 Feb;18(2):229-234. doi: 10.5811/westjem.2016.12.32850.

12. Occupational Employment Statistics. United States Bureau of Labor Statistics. http://www.bls.gov/oes/current/oes_stru.htm\#290000. Accessed July 5, 2016.

13. Wu K, Shepherd J, Jackson S, Chew C. FDA drug safety podcasts: resources for drug information. JAPhA. 2013;53:188-192. doi: 10.1331/JAPhA.2013.12120.

14. Andrejco K, Lowrance J, Morgan B, Padgett C, Collins S. Social media in nurse anesthesia: a model of a reproducible educational podcast. AANA Journal. 2017;85(1):10-16. 